\title{
Normal Chest X-Ray in Patients with Pulmonary Embolism May Involve Increased Risk of Massive Pulmonary Embolism
}

\author{
Hatice Kılıç, Habibe Hezer, Berker Öztürk, Muhammed Sait Besler, Hüseyin Çetin, Hatice Canan \\ Hasanoğlu, Ayşegül Karalezli
}

\section{ABSTRACT}

Purpose: Chest radiography is normal in approximately $20-40 \%$ of acute pulmonary embolism (PE) patients without cardiopulmonary disease. The aim of this study was to determine whether there is any difference between the patients with normal chest $\mathrm{X}$-ray and those with pathological findings in terms of clinical severity and prognosis.

Methods: 178 of PE patients were included in the study. 110 patients had no parenchymal pathology, whereas group $1(n=110)$; group $2(n=68)$ had various pathological parenchymal findings in 68 patients. Clinical and radiological parameters were compared between these groups. Following the diagnosis of PE, the cases were recorded in the fifth year.

Results: In 178 participants; those with normal chest X-ray (group 1), with parenchymal pathological findings on the chest X-ray (group 2); echocardiographic systolic pulmonary artery pressure (sPAP) $(p=0.68)$, gender $(p=0.9)$ and thrombus type $(p=0.41)$ were similar.

The patients in group 1 were not different in terms of central thrombus detected in computed tomography pulmonary angiogram compared to the patients in group 2; however, the chest radiograph of the patients in group 1 had no parenchymal pathology. Central thrombus group 1, group 2, respectively; $97(89.0 \%), 53(77.9 \%), p=0.07$.

There was no significant difference between the two groups in terms of mortality which was followed up in fifth year $(p>0.05)$.

Conclusions: Normal chest X-ray in PE can determine mortality and may involve increased risk of massive PE.

Keywords: Central thrombosis, chest X-ray, computed tomography, pulmonary embolism.

Submitted : June 2, 2021

Published : January 4, 2022

ISSN: 2593-8339

DOI: 10.24018 /ejmed.2022.4.1.910

H. Kılıç*

Ankara Yıldırım Beyazıt University, School of Medicine, Department of Chest Diseases, Ankara,Turkey.

(e-mail: drhaticeb@yahoo.com)

H. Hezer

Ankara Atatürk City Hospital, Department of Chest Diseases, Ankara, Turkey.

B. Öztürk

Ankara Yıldırım Beyazıt University, School of Medicine, Department of Chest Diseases, Ankara, Turkey.

M. S. Besler

Ankara Atatürk City Hospital, Department of Radiology, Ankara, Turkey.

H. Çetin

Ankara Yıldırım Beyazıt University, School of Medicine, Department of Radiology, Ankara, Turkey.

H. C. Hasanoğlu

Ankara Atatürk City Hospital,

Department of Chest Diseases, Ankara, Turkey.

A. Karalezli

Ankara Atatürk City Hospital, Department of Chest Diseases, Ankara, Turkey.

*Corresponding Author

\section{INTRODUCTION}

Pulmonary embolism (PE) occurs mostly because of thrombus originating from lower extremity deep venous thrombosis. Diagnosis in PE begins with clinical suspicion. The presence of risk factors should be considered as well as initial symptoms and findings. PE should be suspected especially in patients who presented with dyspnea and tachycardia and whose chest X-ray was normal and could not be explained by another disease. According to the results of autopsy studies, it was found that most of the cases did not have PE suspicion before death [1].

In clinical cases suspected from PE, Wells clinical scoring is performed. Wells score 2 and above; are considered as medium and high-risk cases. Posteroanterior (PA) chest Xray and d-dimer tests are planned for the diagnosis of PE. PA chest radiography is normal in approximately $20-40 \%$ of PE patients without cardiopulmonary disease. A normal chest Xray does not exclude the possibility of PE. Chest X-ray findings can be subsegmental atelectasis, pleural-based opacity (Hampton hump), pleural effusion, diaphragm elevation, pulmonary artery enlargement, sudden vascular discontinuation, right ventricular dilatation, local vascular reduction, and transparency (Westermark sign). These findings are not specific to PE [1]-[3]. It was shown that there was no significant difference between the patients with and without PE [4].

In our study, we aimed to investigate whether there is a difference in terms of clinical severity and prognosis between 
the patients with normal findings and with pathological findings on chest X-ray.

\section{Materials And Methods}

Our study was conducted in the hospital; (between 2012 and 2017) a total of $178 \mathrm{PE}$ cases who were hospitalized in the Pulmonary Diseases Clinic were included. Electrocardiography, chest X-ray and serum d-dimer levels were performed to the patients with clinical Wells score 2 or above. Computed tomography (CT) pulmonary angiogram (CTPA) was performed in patients with Wells score 2 and above with the findings supporting the diagnosis of PE. PE diagnosis was verified or excluded. The patients who were diagnosed as PE were hospitalized; the data was scanned retrospectively, and the data was recorded. In our hospital registry system, chest X-ray and CTPA scans were analyzed by one radiologist who was blinded to the original CT report and patients' diagnosis.

All the cases were grouped as low, medium, or high risk clinically according to the Turkish Thoracic Consensus Report and the European Society of Cardiology [1], [5], [6]. Serum d-dimer, pro-brain natriuretic peptide (proBNP) levels; systolic pulmonary artery pressure (sPAP) values, presence of right ventricular dilatation in echocardiography; the chest X-ray findings were recorded. In terms of these values, it was statistically evaluated whether there was a significant difference between the two groups.

Group 1 and group 2 cases were scanned from hospital file records, hospital registry system and death notification system. Following the diagnosis of PE, the cases were recorded up to the fifth year.

\section{Statistical ANAlysis}

Shapiro Wilk test was used to determine the distribution of sPAP variables in both age and echocardiography. Therefore, it was expressed with median and minimum maximum. Gender, type of thrombus and other categorical variables were indicated by number $(\%)$.

The patient groups were compared with the Mann-Whitney $\mathrm{U}$ test for age and sPAP by measured echocardiography. Categorical variables were compared with chi-square tests. The level of statistical significance was accepted as $\mathrm{p}<0.05$. For statistical analysis and calculations, IBM SPSS Statistics 21.0 (IBM Corp. Released 2012. IBM SPSS Statistics for Windows, Version 21.0. Armonk, NY: IBM Corp.) was used.

\section{RESULtS}

178 of acute PE patients were included in the study. The patients were divided into two groups according to the parenchymal pathology on chest X-ray. No parenchymal pathology was present in the patients of group $1(n=110)$. There were various pathological parenchymal findings in 68 patients in group $2(n=68)$. Clinical and radiological parameters were compared between these groups.

The median age was 64 (min-max: 21-90) in group 1 and 71 (min-max: 21-94) in group 2. The age of the group 2 was higher than the group $1(\mathrm{p}=0.02)$. There was no significant difference between the groups in terms of gender and embolism type $(p>0.05)$ (Table I).

The patients in group 1 were not different in terms of the patients who have central thrombus detected in CTPA compared to the patients in group 2 (central thrombi group 1 , group 2, respectively; $97(89.0 \%), 53(77.9 \%), \mathrm{p}=0.07)$ (Table II). Although chest radiographs were normal in group 1 patients, central thrombus was found in more cases than in group 2 in CTPA.

\begin{tabular}{|c|c|c|c|}
\hline & $\begin{array}{c}\text { Group } 1 \\
(\mathrm{n}=110) \\
\mathrm{n}, \%\end{array}$ & $\begin{array}{c}\text { Group } 2 \\
(\mathrm{n}=68) \\
\mathrm{n}, \%\end{array}$ & $\mathrm{p}$ \\
\hline $\begin{array}{l}\text { Age median (min- } \\
\text { max) }\end{array}$ & $64(21-90)$ & $71.5(21-94)$ & 0.02 \\
\hline Gender & & & 0.95 \\
\hline Female & $62(56.4)$ & $38(55.9)$ & \\
\hline Male & $48(43.6)$ & $30(44.1)$ & \\
\hline Embolism type & & & 0.41 \\
\hline High risk & $39(35.5)$ & $18(26.5)$ & \\
\hline Moderate-high risk & $63(57.3)$ & $43(63.2)$ & \\
\hline Low risk & $8(7.2)$ & $7(10.3)$ & \\
\hline $\begin{array}{l}\text { sPAP median (min- } \\
\max )\end{array}$ & $50(0-100)$ & $50(0-90)$ & 0.68 \\
\hline
\end{tabular}

TABLE II: COMPARISON OF RADIOLOGICAL FINDINGS OF PATIENTS WITH NORMAL CHEST RADIOGRAPHS (GROUP 1), WITH PARENCHYMAL PATHOLOGICAL FINDINGS ON THE RADIOGRAPHS (GROUP 2)

\begin{tabular}{cccc}
\hline \hline & $\begin{array}{c}\text { Group 1 } \\
(\mathrm{n}=110)\end{array}$ & $\begin{array}{c}\text { Group } 2 \\
(\mathrm{n}=68)\end{array}$ & $\mathrm{p}$ \\
\hline \hline Chest X-Ray Signs & & & \\
Right ventricle enlargement & $83(75.5)$ & $47(71.2)$ & 0.658 \\
Pleural effusion & $0(0.0)$ & $26(38.2)$ & - \\
Atelectasis & $0(0.0)$ & $25(36.8)$ & - \\
Infiltration & $0(0.0)$ & $12(17.9)$ & - \\
Diaphragm elevation & $0(0.0)$ & $30(44.1)$ & - \\
Infarcts & $0(0.0)$ & $12(17.6)$ & - \\
Cut off sign (sudden & $0(0.0)$ & $4(6.0)$ & - \\
vascular discontinuation) & & & \\
Chest CT Signs & & $53(77.9)$ & 0.076 \\
Thrombus on the main & $97(89.0)$ & $29(42.6)$ & $<0.001$ \\
pulmonary artery & $14(12.8)$ & $34(50.0)$ & $<0.001$ \\
Pleural effusion & $11(10.1)$ & $17(25.0)$ & 0.001 \\
Atelectasis & $7(6.4)$ & $24(35.3)$ & $<0.001$ \\
Infiltration & $3(2.8)$ & $19(27.9)$ & $<0.001$ \\
Diaphragm elevation & $8(7.3)$ & $5(7.4)$ & 0.032 \\
Infarcts & $1(0.9)$ & & \\
Cut off sign &
\end{tabular}

Also, the chest CT parenchymal images of the patients in Group 1 were involved significantly lower infiltration than the group 2; $\mathrm{n}=7(6.4 \%) ; \mathrm{n}=17(25.0) \mathrm{p}<0.001$.

Group 1 and group 2 cases were scanned from file records, hospital registry system and death notification system. Following the diagnosis of PE, the cases were recorded according to mortality in the fifth year. There was no significant difference between the two groups in terms of exitus (Table III).

TABLE III: First MONTH, FIRST AND FIFTH YEAR MORTALIY DATA OF PATIENTS WITH NORMAL CHEST RADIOGRAPHS (GROUP 1), WITH PARENCHYMAL PATHOLOGICAL FINDINGS ON THE RADIOGRAPH (GROUP 2)

\begin{tabular}{llll}
\hline \hline & $\begin{array}{c}\text { Group 1 } \\
(\mathrm{n}=110)\end{array}$ & $\begin{array}{c}\text { Group 2 } \\
(\mathrm{n}=68)\end{array}$ & $\mathrm{p}$ \\
\hline
\end{tabular}

First month exitus No $\quad 54(84.4) \quad 32(84.2)$
1.000 


\begin{tabular}{cccc} 
Yes & $10(15.6)$ & $6(15.8)$ & \\
First year exitus & & & 1.000 \\
No & $43(81.1)$ & $25(80.6)$ & \\
Yes & $10(18.9)$ & $9(19.4)$ & \\
Fifth year exitus & & & 0.090 \\
No & $19(79.2)$ & $4(44.4)$ & \\
Yes & $5(20.8)$ & $5(55.6)$ & \\
\hline \hline
\end{tabular}

\section{DISCUSSION}

Normal chest X-ray in PE may determine clinical severity and may involve increased risk of massive PE.

Chest X-ray findings have been shown to be similar in patients with and without PE [4]. In a study, the detection of hilar enlargement on the chest radiograph were correlated with the finding of right ventricular dysfunction of echocardiography [7]. Similar findings were found in another study [8].

Contrast enhanced spiral CTPA can show thrombus in the pulmonary artery bed; directly up to subsegmental level. Central / lobar thrombi of $97 \%$, segmental thrombosis of $68 \%$, and subsegmental thrombosis of $25 \%$ are shown in multislice CT. As the number of detectors increases $(\geq 4)$, the sensitivity of spiral CT detecting peripheral thrombi in subsegmental, and more distal localizations increases [9]. The incidence of subsegmental thrombus in single-slice CT was $4.7 \%$ and this ratio was found to be $9.4 \%$ in the multislice [10]. The meta-analysis that is involving 2020 patients with multi-detector spiral chest CT; were evaluated. The patients who were without any thrombus on chest CT have followed up for three months. The frequency of PE was found to be $1.2 \%$ and fatal PE was found to be $0.6 \%$ at the end of 3 months [11]. The rate of technically insufficient (nondiagnostic) CT angiography due to motion artifacts or incomplete enhancement of pulmonary vessels is 5-8\% [12]. The efficacy of the CTPA increases with the slice number. For instance, 128 slice CT is better than 64 slice BT. Spiral chest CT with a 128 slice detector was performed to the patients in our study.

There are a few studies on the findings in the PA chest Xray in the case of the presence of thrombus in main pulmonary arteries in CT. In a study of 4175 CTPA cases, PE was detected by two radiologists in 41 studies and data from 34 studies were obtained from them. Intraluminal defect was detected in 31 cases. Embolus localization and the number of emboli were determined. 13 patients were diagnosed with different tests of additional CT scan. Nine patients (29\%) had no pathological findings in 31 patients with PE on chest CT. 14 cases $(45 \%)$ had pleural effusion, 10 cases had pleural infarction and nine cases (29\%) had lobar atelectasis [13]. The most common radiological sign was atelectasis in patients with pathological sign on CTPA group $(n=34$, $50.0 \%$ ) in our study.

Also, in another study, CTPA of 92 patients was examined and $\mathrm{PE}$ was detected in 28 cases. Four patients (14\%) had only peripheral thrombus and 24 patients had both central and peripheral thrombus. In the literature, there is no study evaluating the relationship between chest X-ray lung parenchymal findings and thrombus size on chest CT. In a study parenchymal abnormality was detected in 24 cases with $\mathrm{PE}(86 \%)$; in 56 patients $(84 \%)$ without PE. Atelectasis is the most common finding; in 20 (41\%) patients with PE, 41 (64\%) patients without PE were found. Peripheral infarction was detected in seven (25\%) patients with PE and three (5\%) patients without PE [14]. In another study, a total of 423 patients had PE identified on CT in 3141 patients $(13.5 \%)$ who underwent CT angiography. The incidence of pleural effusion was significantly higher in patients with $\mathrm{PE}$ $(243 / 1220,19.9 \%)$ than that in those without embolism $(180 / 1921,9.4 \%)(\mathrm{P}<0.001)$ [15]. While it was detecting pleural effusion of $14(12.8 \%)$ patients in group 1, 29 of $(42.6 \%)$ patients in group 2 in our study.

Creatinine values should be considered for contrast shooting.

This method should not be preferred in patients with renal insufficiency with a creatinine clearance below $50 \mathrm{~mL} / \mathrm{min}$. In these cases, patients with Wells clinical score were evaluated as moderate and high risk $(>2)$; even if the chest radiography is normal, further examination is needed for the diagnosis of PE. According to the guidelines of the European Society of Cardiology, the impairment of renal function tests in these cases; is associated with a decrease in cardiac output and renal hypoperfusion due to PE. For this reason, ventilation-perfusion scintigraphy (V/Q scan)-Single-photon emission computed tomography (SPECT) should be performed in these limited patients with impaired renal function, history of contrast medium-induced anaphylaxis, strong allergic history, myeloma, paraproteinemia, and in pregnant women. If additional disease is present, the diagnosis of PE should be made by performing electrocardiography and lower extremity doppler ultrasound tests. For lung parenchyma, the presence of indirect PE sign in chest CT is evaluated. Accompanied by all these findings; if the cases are high risk, thrombolytic therapy; if they are low-medium risk, the case is evaluated for anticoagulant treatment. Therefore, in patients with normal chest radiography, giving up the diagnosis of $\mathrm{PE}$ is a wrong approach [6], [11].

Mismatch may not be observed in some cases with V/Q scan-SPECT. Some emboli may not form mismatches since they do not cause vascular occlusion such as saddle emboli [16].

In contrast, a normal perfusion scintigraphy, according to guidelines, excludes PE. However, major non occlusive emboli do not cause any clinical symptoms such as sudden death. Similarly, small emboli causing occlusion are detected and treated by V/Q scan-SPECT [17].

In a study published in the chest with 6674 cases, $40.5 \%$ of the cases had central PTE. Following anticoagulation (2175.4 patient-years), patients with central thrombosis had increased risk of recurrence compared to patients with peripheral thrombus (11.0 vs. 8.0 per 100 patient-years; adjusted hazard ratio [HR] 1.34, confidence interval [CI] 95\% 1.01-1.78) [18]. In our study, there was no significant difference in mortality between the patients with normal chest X-ray and other groups at the time of diagnosis at the first year and fifth year.

In the light of these evidence, a total of 110 patients with normal chest X-ray findings were evaluated in our study. Pulmonary embolism was detected in CTPA in 63 (57.3\%) patients with high-risk embolism in 39 (35.5\%) patients. Also, in 68 patients with parenchymal signs on chest X-ray, 
$18(26 \%)$ patients with high risk and in $43(67 \%)$ patients with moderate high risk were diagnosed with PE by CTPA. Sometimes, in patients with renal dysfunction in emergency departments, chest $\mathrm{CT}$ is performed without contrast agent for the diagnosis of PE. However, as shown in our study, pulmonary parenchymal findings in chest CT may not reveal any signs of thrombus in the main pulmonary arteries. Even planar V/Q scan may not be able to detect perfusion defects in the saddle thrombosis or main pulmonary artery thrombi [16]. For this reason, more attention should be paid in cases with no parenchymal findings and renal impairment. There are some limitations in PE studies. The diagnosis of this acute disease should be made according to the clinical findings of the patient.

The limitation of the study is that the laboratory parameters are not compared according to the type of embolism (central or peripheral).

\section{CONCLUSION}

In conclusion, a patient pre diagnosed with PE having a normal chest X-ray may have an increased risk of massive PE. Even if the chest X-ray is completely normal, a PE with high-risk central thrombus may be found by CTPA. In our study, it was also observed that normal chest X-ray may not be a prediction of good prognosis.

\section{ETHICAL STATEMENT}

All procedures performed in studies involving human participants were in accordance with the ethical standards of the institutional and/or national research committee (Ankara Yildirim Beyazit University, Faculty of Medicine, local ethics council, 28.05.2018/119) and with the 1964 Helsinki declaration and its later amendments or comparable ethical standards. Informed consent was obtained from all individual participants included in the study.

\section{CONTRIBUTION OF AUTHORS}

Substantially contributed to the conception of the work: $\mathrm{HK}, \mathrm{AK}, \mathrm{HCH}$. Writing and revision of the manuscript: HK, AK. Data verification: $\mathrm{HK}, \mathrm{HH}, \mathrm{BO}, \mathrm{HC}$. Approved the final version: $\mathrm{HK}, \mathrm{AK}, \mathrm{HCH}$. Authors declares no conflict of interest.

\section{REFERENCES}

[1] Chen S, Mulgrew B, Grant PM. A clustering technique for digital communications channel equalization using radial basis function networks. IEEE Trans. on Neural Networks. 1993; 4: 570-578.

[2] Stein PD, Terrin ML, Hales CA, Palevsky HI, Saltzman, HA, Thompson BT, et al. Clinical, laboratory, roentgenographic, and electrocardiographic findings in patients with acute pulmonary embolism and no pre-existing cardiac or pulmonary disease. Chest. 1991; 100(3): 598-603.3.

[3] Worsley DF, Alavi A, Aronchick JM, Chen JT, Greenspan RH, Ravin CE. Chest radiographic findings in patients with acute pulmonary embolism: observations from the PIOPED Study. Radiology. 1993; 189(1): 133-136

[4] Powell T, Müller NL. Imaging of acute pulmonary thromboembolism: should spinal computed tomography replace the ventilation perfusion scan. Clin Chest Med. 2003; 24: 29-38.
[5] Kearon C, Akl EA, Ornelas J, Blaivas A, Jimenez D, Bounameaux H, et al. Antithrombotic therapy for VTE disease: CHEST guideline and expert panel report. Chest. 2016; 149(2): 315-352.

[6] Konstantinides SV, Torbicki A, Agnelli G, Danchin N, Fitzmaurice D, Galie N, et al. 2014 ESC Guidelines on the diagnosis and management of acute pulmonary embolism: The Task Force for the Diagnosis and Management of Acute Pulmonary Embolism of the European Society of Cardiology (ESC) Endorsed by the European Respiratory Society (ERS). European Heart Journal. 2014; 35: 3033-73.

[7] Abul Y, Ozsu S, Karakurt S, Ozben B, Durmus I, Toprak A, et al. Prediction of right ventricular dysfunction from radiographic estimates of right descending pulmonary artery in hemodynamically stable pulmonary embolism patients. Cardiology Journal. 2013; 20(2): 184189.

[8] Pollack CV, Schreiber D, Goldhaber SZ, Slattery D, Fanikos J, O'Neil $\mathrm{BJ}$, et al. Clinical characteristics, management, and outcomes of patients diagnosed with acute pulmonary embolism in the emergency department: initial report of EMPEROR (Multicenter Emergency Medicine Pulmonary Embolism in the Real-World Registry). Journal of the American College of Cardiology. 2011; 57(6): 700-706.

[9] Mos ICM, Klok FA, Kroft LJM, De Roos A, Dekkers OM, Huisman MV. Safety of ruling out acute pulmonary embolism by normal computed tomography pulmonary angiography in patients with an indication for computed tomography: systematic review and metaanalysis. Journal of Thrombosis and Haemostasis. 2009; 7(9): 14911498.

[10] Carrier M, Righini M, Wells PS, Perrier A, Anderson DR, Rodger MA, et al. Subsegmental pulmonary embolism diagnosed by computed tomography: incidence and clinical implications. A systematic review and meta-analysis of the management outcome studies. Journal of Thrombosis and Haemostasis. 2010; 8(8): 1716-1722.

[11] Torbicki A, Perrier A, Konstantinides S, Agnelli G, Galiè N, Vachiery JL. Guidelines on the diagnosis and management of acute pulmonary embolism: the Task Force for the Diagnosis and Management of Acute Pulmonary Embolism of the European Society of Cardiology (ESC). European Heart Journal. 2008; 29(18): 2276-2315.

[12] Merten GJ, Burgess WP, Gray LV, Holleman JH, Roush TS, Kowalchuk GJ, et al. Prevention of contrast induced nephropathy with sodium bicarbonate: a randomized controlled trial. JAMA. 2004; 291: 2328-34.

[13] Johnson PT, Wechsler RJ, Salazar AM, Fisher AM, Nazarian LN, Steiner RM. Spiral CT of Acute Pulmonary Thromboembolism: Evaluation of Pleuroparenchymal Abnormalities Journal of Computer Assisted Tomography. 1999; 23(3): 369-373.

[14] Shah AA, Davis SD, Gamsu G, Intriere L. Parenchymal and Pleural Findings in Patients with and Patients without Acute Pulmonary Embolism Detected at Spiral CT. Radiology. 1999; 211(1): 147-53.

[15] Liu M, Cui A, Zhai ZG, Guo XJ, Li M, Teng LL, et al. Incidence of Pleural Effusion in Patients with Pulmonary Embolism. Chinese Medical Journal. 2015; 128: 1032-6.

[16] Schumichen C. V/Q-scanning/SPECT for the diagnosis of pulmonary embolism. Respiration. 2003; 70: 329-342.

[17] Bajc M, Neilly B, Miniati M, Mortensen J, Jonson B. Methodology for Ventilation/Perfusion SPECT. Seminars in Nuclear Medicine 2010; 40(6): 415-425.

[18] Gouin B, Blondon M, Jimenez D, Fernandez-Capitan C, Bounameaux $\mathrm{H}$, Soler S, et al. Clinical prognosis of nonmassive central and noncentral pulmonary embolism: a register-based cohort study. Chest. 2016; 151(4): 829-837. 